\title{
A Simple Technique to Fabricate Broadrick's Occlusal Plane Analyzer for Full Mouth Rehabilitation of a GERD Patient: A Clinical Report
}

\section{Dewan $\mathrm{H}^{*}$}

Department of Prosthetic Dental Sciences, Jazan University, Kingdom of Saudi Arabia

*Corresponding author: Harisha Dewan, Assistant Professor, Department of Prosthetic Dental Sciences, Jazan University, Jazan, Kingdom of Saudi Arabia, Tel: +966554914588; Email: harisha.dewan@yahoo.com

Case Report

Volume 4 Issue 3

Received Date: August 13, 2019

Published Date: August 20, 2019

DOI: 10.23880 /oajds- 16000237

\section{Abstract}

This case report discusses the phases of prosthodontic rehabilitation, from diagnosis to final treatment, of a 38-year-old female (bruxer) with the past medical history of GERD. Stomach acid regurgitating into the esophagus or oral cavity can lead to a chronic condition known as the Gastroesophageal reflux disease (GERD) which is often a prominent cause of heartburn. The two common oral manifestations of GERD are tooth erosion and wear. There was a loss of anterior guidance and subsequent reduction of the vertical dimension. Increasing the vertical dimension was planned using an occlusal splint. Once the adaptability of the new vertical dimension had been confirmed, the final reconstruction was initiated. When planning multiple long span posterior restorations, a properly constructed occlusal plane is an essential consideration. A simple occlusal plane analyzer has been customized and used to assist the operator in the development of an initial mandibular occlusal plane.

Keywords: Worn dentition, Vertical dimension, Occlusal splint

\section{Abbreviations: GERD: Gastroesophageal Reflux} Disease.

\section{Introduction}

Wear of tooth surfaces can be due to attrition, abrasion, and erosion depending on its cause. In many situations, there exists a combination of these processes, because of which, a differential diagnosis is not always possible [1]. Excessive occlusal wear can lead to pulpal pathosis, occlusal disturbances, improper function, and esthetic disfigurement [2]. Therefore, it is important to identify the causative factors contributing to the wear and to evaluate VDO changes caused by the same [3].

As teeth are worn, the alveolar bone undergoes an adaptive process and maintains the VDO by compensating for the loss of tooth structure. Therefore, VDO should be conservative and should be changed only with a careful approach, [4,5] especially, in bruxers where increasing the VDO puts an overload on the remaining teeth which generally leads to the destruction of the restorations or, in some cases, teeth themselves [4]. 


\section{Open Access Journal of Dental Sciences}

However, the rehabilitation of the severely worn dentition becomes a challenge when the space for restoration is insufficient. In 1975, Dahl, et al. [6] studied the use of an anterior removable cobalt-chromium occlusal device to an 18-year-old patient with severe localized attrition to generate interocclusal space for subsequent restoration. The tooth movement occurred involving a combination of orthodontic anterior teeth intrusion and eruption of the posterior teeth. Future observations of this treatment reported that the vertical relations were practically quite stable $[7,8]$. Nowadays the use of the adhesive resin [9] or an overlay splint [10] instead of a cobalt-chromium device has replaced the technique.

Management of severely worn dentition using fixed prostheses requires proper management of occlusal plane [11]. Assessment of the vertical dimension is important for the management, and a careful comprehensive treatment plan is required for each case [12]. Study casts mounted on the articulator with the diagnostic wax-up done, can provide us with important information for proper treatment planning. Tolerance of changes to the vertical dimension of occlusion is usually confirmed with the clinical evaluation of the patient having a splint or provisional prosthesis [13].

This clinical report describes the treatment of a patient who was clinically monitored to evaluate the adaptation to the removable occlusal splint during a 1 month trial period and the provisional restorations for 3 months $[14,15]$.

\section{Case Report}

A 38 years old Saudi unmarried female reported to the College of Dentistry, Jazan University, with the chief complaint of broken-down teeth. The patient gave a medical history of acid reflux (GERD) for the past four years, anxiety and stress. Tooth erosion and wear are common oral manifestations of GERD. She was taking omeprazole $20 \mathrm{mg}$ and hyoscine butylbromide $10 \mathrm{mg}$ once daily.

Intraoral examination spotted a generalized loss of tooth structure. The severe tooth wear was attributed to parafunctional habits, unsuitable restorations, and posterior dental interferences (Figure 1A). The case was diagnosed with generalized chronic gingivitis (plaqueinduced) with occlusal caries in 18, 12, 21, 22, 28, 47; cervical caries in $14,33,34,43,44$ and secondary caries in 11. The treatment planning included oral prophylaxis (scaling ,polishing and root planning), oral hygiene instructions and motivation of the patient, class I restorations in $18,12,21,22,28,47$, class IV restorations in 11 , class $\mathrm{V}$ restorations in $33,34,43,44,14$ followed by reevaluation. The patient's interocclusal rest space that was measured between the nose tip and chin tip was 5 $\mathrm{mm}$ which was greater than the normal value, $2-4 \mathrm{~mm}$. Also, the increased freeway space altered /s/ sound to $/ \mathrm{J} /$ [5]. It was decided to increase the vertical dimension by 1 $\mathrm{mm}$. As there was a clinical evaluation of reduced VDO, full mouth zirconia crowns from 17 to 27 and 37 to 47 , followed by night guard with an increase in VDO was planned with periodic recall.

Scaling and root planning was done with an emphasis on a healthy diet and oral hygiene instruction. Class I, IV, $\mathrm{V}$ cavity preparation was done and composite restoration in $18,14,12,11,21,22,28,33,34,43,44$ and 47 were given. The method of increasing VDO (about $1 \mathrm{~mm}$ ) with the splint was used to determine the desirable VDO of the fixed interim prostheses (Figures 1B \& C). The patient's comfort to the increased VDO was checked and reevaluated during one month trial period. No muscle or temporomandibular tenderness was found.

Before making the final impression, diagnostic plaster casts (Moldano Dental Stone, Bayer Co., Leverkusen, Germany) were made from alginate impressions (Tropicalgin, Zhermack, Badia Polesine, Rovigo, Italy). An acrylic anterior deprogramming device fabricated from pattern resin (Pattern Resin, LS, GC America, ALSIP, IL, USA) was used along with baseplate wax (Cavex Setup Regular Modelling Wax, Cavex Holland BV, Haarlem, Netherlands) to record the bite registration. This record along with an arbitrary facebow was used to mount the casts on the Whip mix articulator (The condylar guidance on the articulator was set at an average value of 35 degrees). The curves of Spee and Wilson as well as the orientation of the occlusal plane were determined using a Broadrick's occlusal plane analyzer [11,16] (Figure 2). The Broadrick's flag, commonly used, is an extremely useful tool in the construction of the curve of Spee in harmony with anterior condylar guidance and thus allowing posterior disclusion when the mandible is protruding. The mandibular incisors are, in result, set in an accurate functional and esthetic position. Rectangular cardboard (18 $\mathrm{cm} \times 12 \mathrm{~cm}$ approximately) was taken and a white sheet of the same measurement was attached to the cardboard with the help of paper clips. The whole assembly was fixed to the top slot of the Whip mix articulator with help of putty impression material. The distal slope of the lower canine tooth was chosen as the 
anterior survey point from where a compass with a fourinch radius was used to get a long arc on the sheet, marked as the anterior survey line. The distal slope of the distobuccal cusp of the lower second molar was the posterior survey point and a short arc from this point was drawn on the sheet to intersect the earlier marked anterior survey line (Figure 2). The two survey lines bisect at the central survey point. Now the point of the compass was placed at the central survey point, and a 4inch radius was drawn passing through the buccal surfaces of the mandibular teeth which would, finally, decide the future occlusal plane in this patient.

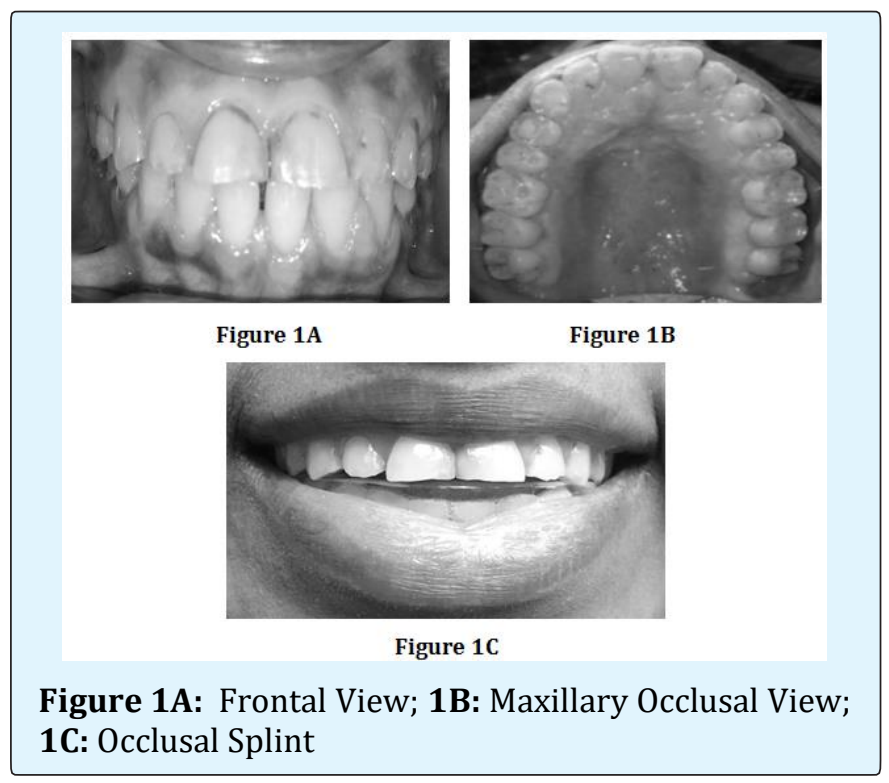

Figure 1A: Frontal View; 1B: Maxillary Occlusal View;

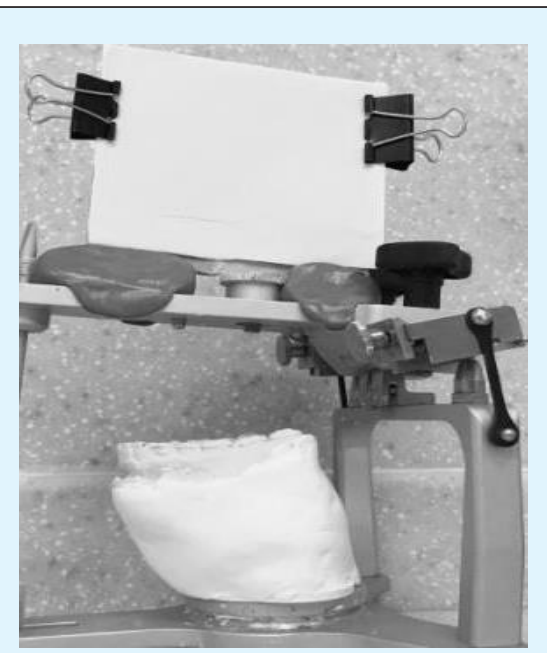

Figure 2: Customized Broadrick's occlusal plane analyzer to know the posterior plane of new crowns and curve of Spee.
After diagnostic wax-up at new OVD (Figure 3), a new cast was made (with duplication of diagnostic wax-up) and via auto polymerizing acrylic resin (Tempron, GC Europe), provisional crowns were fabricated using a vacuum formed matrix (Drufolen $\mathrm{H}$; Dreve Dentamid $\mathrm{GmbH}$, Unna, Germany) using this cast. Intraoral composite mockup with the increased size of anteriors was done and shown to the patient. Teeth preparation was carried out using a putty index which was made with the help of the diagnostic wax-up. The provisional fixed restorations were cemented with temporary cement (FREEGENOL TEMPORARY PACK; GC Corp., Tokyo, Japan), and the patient's adaptation was monitored (Figure 4). The waiting period was of 3 months with regular recall in between after every 15 days. For three months, the interim restorations were adjusted and used as a guide for the definitive oral rehabilitation. During this period, muscle tenderness, the discomfort of TMJ, the problem in mastication, restricted mandibular movements, problems in swallowing, and speech were evaluated. Slow improvement in speech, mastication, and esthetics confirmed the patient's acceptance of the new mandibular position with the newly restored VDO. The anterior guidance and posterior teeth disclusion on excursive movements were established. Alginate impression with the temporary crowns in the mouth was made and the cast was mounted in centric relation using facebow to verify and copy the same condylar guidance. The already adjusted occlusion scheme was transferred to a customized anterior guide table, which was made with acrylic resin (PATTERN RESIN; GC Corp, Tokyo, Japan). Final preparation was performed and definitive impressions were made with polyvinylsiloxane impression material (Extrude; Kerr Corp., Romulus, Germany). The next step was to record the unilateral bite registrations using bite registration material (StoneBite; Dreve Dentamid GmbH, Unna, Germany) and cross mounting was done. Zirconia coping trial was done. Final porcelain restorations were made using a customized anterior guide table and cemented with resin-modified glass ionomer cement (FujiCEM; GC America, Alsip, USA) (Figure 5). Because the patient's anterior guidance table was used in the production of definitive restoration, the amount of occlusal adjustment on the lingual surface of maxillary anterior teeth was minimum. Canine guidance was verified (Figure 6). Oral hygiene instructions and regular check-ups were administered. A hard acrylic resin full occlusal splint (Acropars, Marlic Co., Tehran, Iran) was fabricated for night use to prevent parafunctional occlusal wear. Minor adjustments were required at first post insertion visit. 

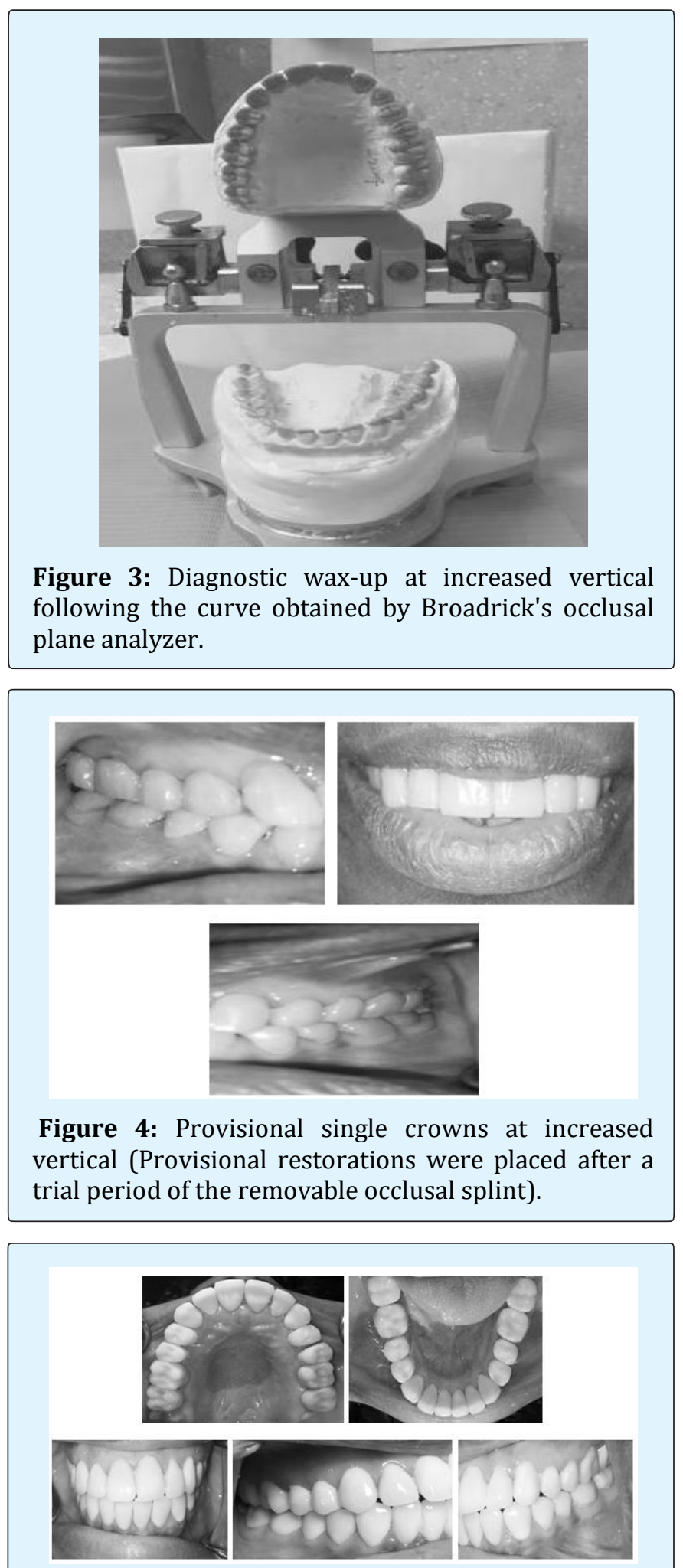

Figure 5: Definitive restorations were delivered.

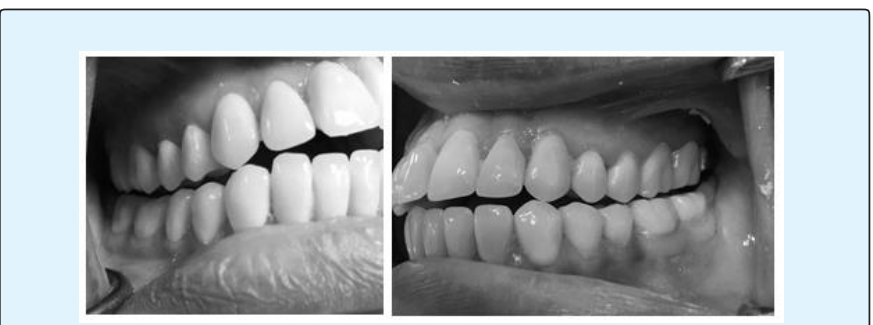

Figure 6: Canine guidance achieved on both the sides.

\section{Discussion}

The multifactorial aetiology and uncertainty of the long-term outcomes of treatment methods and materials used in generalised worn out cases cause difficulty in formulating a proper treatment plan. In this case, due to the loss of palatal tooth structure on maxillary anterior teeth, minimal preparation on the palatal surfaces of maxillary anterior teeth was indicated. Composite resin was used to bond the anterior restorations due to its higher acidic resistance and stronger bonding strength. Resin-modified glass ionomer was used to cement the other restorations due to its lower technique sensitivity and acidic resistance.

An occlusal plane analyzer assists the operator in the development of an initial mandibular occlusal plane in diagnostic casts and also important in guiding the operator in actual tooth preparations and achieving proper contour in the final restorations $[11,16]$. The curve of Spee may be pathologically altered in situations like the present one. Restoration of the dentition to such an altered occlusal plane can introduce posterior protrusive interferences [4] causing abnormal activity in mandibular elevator muscles, especially the masseter and temporalis muscle [5].

The most important advantages of zirconia restorations are esthetics and strength, making it our choice for the definitive restorations [17,18]. A mandibular protective splint with a medium thermoplastic sheet was fabricated and given to the patient to prevent further tooth damage due to bruxism.

\section{Conclusion}

In this clinical report, a comprehensive study and practical approach using a simple, customized Broadrick's occlusal plane analyser were directed towards reconstruction, restoration, and maintenance of the health of the entire oral mechanism. 


\section{Open Access Journal of Dental Sciences}

\section{Acknowledgement}

The author would like to extend gratitude to Jazan University, College of Dentistry, for giving permissions and providing the required materials to complete this case.

\section{References}

1. Singh A, Bhede R, Chandak M, Saxena A (2013) Full Mouth Rehabilitation a Multidisciplinary Approach: CaseReport. Journal of Dental and Medical Sciences 9(2): 65-69.

2. Turner KA, Missirlian DM (1984) Restoration of the extremely worn dentition. J Prosthet Dent 52(4): $467-$ 474.

3. Prasad S, Kuracina J, Monaco EA (2008) Altering occlusal vertical dimension provisionally with base metal onlays: a clinical report. J Prosthet Dent 100(5): 338-342.

4. Dawson PE (2008) Functional Occlusion-From TMJ to smile design. $1^{\text {st }}$ (Edn.), New York: Elsevier Inc., pp: 430-452.

5. Abdou J (2012) Safety of increasing vertical dimension of occlusion: a systematic review. Quintessence Int 43(5): 369-380.

6. Dahl BL, Krogstad O, Karlsen K (1975) An alternative treatment in cases with advanced localized attrition. J Oral Rehabil 2(3): 209-214.

7. Dahl BL, Krogstad O (1985) Long-term observations of an increased occlusal face height obtained by a combined orthodontic/prosthetic approach. J Oral Rehabil 12(2): 173-176.

8. Dahl BL (1995) The face height in adult dentate humans, A discussion of physiological and prosthodontic principles illustrated through a case report. J Oral Rehabil 22(8): 565-569.

9. Hemmings KW, Darbar UR, Vaughan S (2000) Tooth wear treated with direct composite restorations at an increased vertical dimension: results at 30 months. J Prosthet Dent 83(3): 287-293.

10. Sato S, Hotta TH, Pedrazzi V (2000) Removable occlusal overlay splint in the management of tooth wear: a clinical report. J Prosthet Dent 83(4): 392395.

11. Chaturvedi S, Verma AK, Ali M, Shah M (2012) Full mouth rehabilitation using a custom-made broadrick flag: A case report. International Journal of Case Reports and Images 3(5): 41-44.

12. Areej Darraj, Khurshid A Mattoo (2017) Full Mouth Rehabilitation Involving Occlusal Plane CorrectionCase Report. JMSCR 5(9): 28204 -28208.

13. Song MY, Park JM, Park EJ (2010) Full mouth rehabilitation of the patient with severely worn dentition: a case report. J Adv Prosthodont 2(3): 106110.

14. Yunus N, Abdullah H, Hanapiah F (2001) The use of implants in the occlusal rehabilitation of a partially edentulous patient: a clinical report. J Prosthet Dent 85(6): 540-543.

15. Ganddini MR, Al-Mardini M, Graser GN, Almog D (2004) Maxillary and mandibular overlay removable partial dentures for the restoration of worn teeth. J Prosthet Dent 91(3): 210-214.

16. Gupta R, Luthra RP, Hardik, Sheth H (2019) Broadrick's occlusal plane analyzer: A review. International Journal of Applied Dental Sciences 5(1): 95-98.

17. Edwin Ruales, Engler MLPD, Vaz P, Özcan M, Volpato CAM (2018) Esthetic and functional rehabilitation of bilateral congenital absence of maxillary lateral incisors: Minimally invasive surgical and prosthetic approach. J Esthet Restor Dent 31(1): 5-12.

18. Agustin PR, Juan LRR, Alberto F, María FSR, Antonio FF (2014) Zirconia in fixed prosthesis, A literature review. J Clin Exp Dent 6(1): 66-73. 